\title{
Aktuelle Rechtsprechung zum österreichischen und europäischen Urheberrecht
}

\author{
Manfred Büchele
}

\section{EuGH C-160/15, GS Media ${ }^{1}$}

Die TV-Moderatorin Britt Dekker ließ sich für den niederländischen Playboy ablichten. ${ }^{2}$ Die Nutzungsrechte an den Fotos wurden exklusiv der Verlegerin des Playboy, Sanoma, eingeräumt.

Die beklagte GS Media betreibt den Blog GeenStijl, in dem ein Artikel über Frau Dekker veröffentlicht wurde. Der Artikel enthielt einen Link, über den Fotos aus dem Shooting abrufbar waren, für den aber keine Zustimmung von Sanoma vorlag. Sanoma forderte GS Media auf, den Link zu entfernen, was von GS Media ignoriert wurde. Stattdessen veröffentlichte GS Media einen weiteren Artikel, in dem wiederum ein Link zu den Fotos von Frau Dekker enthalten war. Samona klagte daraufhin die GS Media.

Der Oberste Gerichtshof der Niederlande legte dem EuGH die Frage zur Vorabentscheidung vor, ob - zusammengefasst - Links auf fremde unrechtmäßig veröffentlichte Inhalte zulässig sind. ${ }^{3}$

\footnotetext{
${ }^{1}$ EuGH 08.09.2016, C-160/15, GS Media, ÖB1 2017/15, 56 (Handig) = MR-Int 2017, 23 (Walter) = ecolex 2016/478, 1087 (Zemann).

${ }^{2}$ Das Fotoshooting fand im Oktober 2011 statt; der Playboy veröffentlichte die Fotos von Frau Dekker im Dezember 2011.

${ }^{3}$ Die EuGH-Entscheidungen C-466/12, Svensson/Retriever Sverige, ÖB1 2014/33 (Handig) $=$ MR 2014, 27 (Walter) = ecolex 2014/141 (Büchele) und C-348/13, BestWater, MR-Int 2014, 120 (Walter) konnten vorliegend nicht fruchtbar gemacht werden, weil diese darauf gründeten, dass die verlinkten Werke mit Zustimmung der Rechteinhaber veröffentlicht worden waren.
}

Beim 1. Bozner Wirtschaftsrechttag zum italienischen, europäischen und internationalen Immaterialgüterrecht am 15.06.2018 gehaltener Vortrag.

\footnotetext{
M. Büchele ( $\square)$

Institut für Unternehmens- und Steuerrecht, Universität Innsbruck, Innsbruck, Österreich

E-Mail: Manfred.Buechele@uibk.ac.at
} 
Der EuGH bildet drei Fallgruppen, deren innere Verbindung vornehmlich darin besteht, subjektive Elemente in ein grundsätzlich objektives Tatbestandsmerkmal einzubeziehen: ${ }^{4}$

1. Der Linksetzer handelt in Gewinnerzielungsabsicht beziehungsweise zu Erwerbszwecken. ${ }^{5}$ Dann gilt die widerlegbare Vermutung, dass der Linksetzer vom Schutz des Werks und von der fehlenden Zustimmung des Rechteinhabers wusste. Von ihm kann erwartet werden, dass er die erforderlichen Überprüfungen vornimmt. ${ }^{6}$ Liegen diese Umstände vor, dann ist der Link eine öffentliche Wiedergabe, die vom Rechteinhaber zu genehmigen ist.

$\rightarrow$ Im Ergebnis fällt GS Media in diese Kategorie, das heißt GS Media hätte die Fotos nur mit Zustimmung verlinken dürfen, weil sie eine öffentliche Wiedergabe vornimmt.

Die anderen beiden Fallgruppen, die gegenständlich nicht relevant waren:

2. Der Linksetzer handelt nicht in Gewinnerzielungsabsicht, er hat aber positive Kenntnis oder grob fahrlässige Unkenntnis in Bezug auf die Rechtswidrigkeit der Veröffentlichung. ${ }^{7}$

$\rightarrow$ Auch dann ist der Link eine öffentliche Wiedergabe, welche vom Rechteinhaber zu genehmigen ist.

3. Die dritte Kategorie: Der Linksetzer handelt nicht in Gewinnerzielungsabsicht und er hat auch keine positive Kenntnis oder grob fahrlässige Unkenntnis in Hinblick auf die fehlende Zustimmung des Rechteinhabers zur Veröffentlichung.

$\rightarrow$ In diesem Fall verwirklicht der Link keine öffentliche Wiedergabe. ${ }^{8}$

\section{EuGH C-527/15, Stichting Brein/Filmspeler 9}

Das Streamen von Multimedia-Inhalten ist sehr beliebt. Am Markt werden dafür verschiedene Hard- und Software-Lösungen angeboten.

Der Filmspeler ist eines dieser Abspielgerät für Filme, Serien, Musik, für Liveübertragungen und so weiter. Auf ihm waren Add-Ons vorinstalliert, wobei diese Add-Ons vorwiegend Links auf Filme und Serien enthielten - vor allem Links

\footnotetext{
${ }^{4}$ Ähnlich Schmidt-Wudy in seiner Glosse zu EuGH 08.09.2016, C-160/15, GS Media, EuZW 2016, 785 (789 f).

${ }^{5}$ Weder die - vom Rechteinhaber zu bescheinigende oder gar nachzuweisende - Gewinnerzielungsabsicht noch die Erwerbszwecke werden vom EuGH näher definiert.

${ }^{6}$ Der Umfang der Prüfpflichten des Linksetzers ist offen, meines Erachtens besteht aber keine nachfolgende Überwachungspflicht.

${ }^{7}$ Zum Beispiel durch einen Hinweis des Rechteinhabers, weil es der Rechteinhaber ist, der nachweisen beziehungsweise zumindest glaubhaft machen muss, dass der Linksetzer vorsätzlich oder grob fahrlässig handelt.

${ }^{8}$ Außer der Link umgeht eine sogenannte beschränkende Maßnahme, beispielsweise eine Aboschranke.

${ }^{9} \mathrm{EuGH}$ 26.04.2017, C-527/15, Stichting Brein/Filmspeler, ÖB1-LS 2017/25, 238 (Handig) = MRInt 2017, 33 (Walter) $=$ ecolex 2017/325, 790 (Zemann) $=$ jusIT 2017/44, 94 (Staudegger) .
} 
auf solche Inhalte, die rechtswidrig online zugänglich waren. Davon abgesehen hat der Beklagte den Filmspeler nicht nur vertrieben, sondern auch damit beworben, dass die Nutzer die Inhalte kostenlos und äußerst einfach abrufen können, ohne sich um die Erlaubnis der Rechteinhaber kümmern zu müssen.

Die Stichting Brein aus den Niederlanden klagte, wobei sie ganz generell den Verkauf des Filmspelers beanstandete. ${ }^{10}$

Für den EuGH war bei der öffentlichen ${ }^{11}$ Wiedergabe entscheidend, dass die vorinstallierten Add-Ons und die in den Add-Ons enthaltenen Links auf eindeutig illegale Streaming-Angebote zielten. ${ }^{12}$

Wenn der Beklagte in Erwerbsabsicht, in Gewinnerzielungsabsicht, für den Filmspeler wirbt und dabei ausdrücklich betont, dass mit dem Filmspeler Inhalte abgerufen werden können, für die keine Erlaubnis der Rechteinhaber vorliegt, dann besteht die Vermutung, dass der Beklagte von der fehlenden Erlaubnis der Rechteinhaber wusste.

Dieses Argument - dass in den vorinstallierten Add-Ons Links auf unzulässige Quellen enthalten waren und dass der Beklagte davon wusste - überträgt der EuGH in einer Art Reflex auf das Gerät, auf den Filmspeler an sich.

Das heißt: Schon der Verkauf des Filmspelers mit den vorinstallierten Add-Ons verwirklichte eine Wiedergabe, die vom Rechteinhaber genehmigt werden musste. ${ }^{13}$ Ohne die Add-Ons hätte man den Filmspeler durchaus verkaufen können, wie zum Beispiel auch den Fire TV-Stick oder Chromecast oder jedes Smartphone. Sie alle greifen nicht ins Recht der öffentlichen Wiedergabe ein, solange man die entsprechenden Add-Ons nicht installiert.

Für die Käufer des Filmspelers, die beim Streamen vorübergehende Vervielfältigungen anfertigen, gilt Folgendes: Wenn die Käufer ,freiwillig und in Kenntnis der Sachlage", sprich wenn sie bewusst auf illegale Streaming-Quellen zugreifen, ist die Ausnahme für flüchtige und begleitende Vervielfältigungen ${ }^{14}$ nicht anwendbar. Die freie Werknutzung für vorübergehende Vervielfältigungen greift in diesem Fall nicht, weil sich die Nutzer nicht auf ihre Unkenntnis berufen können.

Im Ergebnis untersagt der EuGH den Verkauf des Filmspelers.

\footnotetext{
${ }^{10}$ Die Stichting Brein stützte ihre Klage nicht auf bestimmte verletzte Werke. Mit Blick auf das Wettbewerbsrecht fällt auf, dass auch und gerade dort ein Wettbewerber nicht die Verletzung eines subjektiven Rechts geltend machen und auch nicht nachweisen muss, welches konkrete Produkt vom Wettbewerbsverstoß betroffen ist.

${ }^{11}$ Quantitativ setzt die öffentliche Wiedergabe eine grundsätzlich unbestimmte und recht große Zahl von Adressaten voraus; qualitativ erfordert sie die Wiedergabe entweder durch ein anderes technisches Verfahren oder an ein neues Publikum, das der Rechteinhaber mit seiner Zustimmung zur ursprünglichen Wiedergabe nicht erfassen wollte.

${ }^{12}$ Hyperlinks geben die verlinkten Inhalte nicht in einem anderen technischen Verfahren wieder.

${ }^{13}$ Dieser vom EuGH zugestandene Vorfeldschutz ist nicht selbstverständlich, weil Erwägungsgrund 27 Info-RL (Richtlinie 2001/29/EG des Europäischen Parlaments und des Rates vom 22. Mai 2001 zur Harmonisierung bestimmter Aspekte des Urheberrechts und der verwandten Schutzrechte in der Informationsgesellschaft, AB1 L 167 vom 22.06.2001, 10) davon ausgeht, dass das bloße Bereitstellen einer Einrichtung, die eine Wiedergabe ermöglicht, selber keine Wiedergabe ist. ${ }^{14}$ Vergleiche $§ 41 \mathrm{a}$ UrhG.
} 


\section{OGH 4 Ob 121/17y, BitTorrent ${ }^{15}$}

Der Entscheidung BitTorrent lag folgender Sachverhalt zugrunde:

Die Antragstellerin im Sicherungsverfahren war eine Verwertungsgesellschaft, die die Rechte von Schallträgerherstellern und ausübenden Künstlern wahrnahm. Die Antragsgegnerinnen waren Access-Provider, die den Zugang zu The Pirate Bay vermittelten.

Die Nutzer, die Kunden der Access-Provider, konnten entsprechende Inhalte über The Pirate Bay herunterladen und verteilen, und zwar mit einem BitTorrent-Client. Der OGH hatte der Frage nachzugehen, ob die Access-Provider den Zugang zu The Pirate Bay blockieren mussten - die Entscheidung handelt somit von Zugangssperren.

Laut OGH dürfen die Access-Provider zu Zugangssperren verpflichtet werden. ${ }^{16}$ Die Sperren müssen aber verhältnismäßig sein und ein angemessenes Gleichgewicht zwischen den Grundrechten wahren. ${ }^{17}$

Wenn die Zugangssperren nicht nur unrechtmäßige, sondern auch rechtmäßige Inhalte erfassen (sogenanntes Overblocking), kommt es nicht nur auf das Mengenverhältnis zwischen rechtmäßigen und unrechtmäßigen Inhalten an, sondern es müssen auch andere Merkmale berücksichtigt werden. ${ }^{18}$ Im gegenständlichen Fall war das unproblematisch, weil der Antragstellerin der Anscheinsbeweis gelungen war, dass auf The Pirate Bay nahezu ausschließlich (Magnet-)Links auf unzulässige Inhalte gesammelt, aufbereitet und angeboten wurden (und werden). ${ }^{19}$

Im Ergebnis müssen die Access-Provider den Zugang zu The Pirate Bay blockieren.

\section{OGH 3 Ob 1/18w, Impugnationsklage bei Sperrverfügungen/UPC Telekabel III ${ }^{20}$}

In engem Zusammenhang mit dem Sperren von Websites steht die Frage, was den Access-Providern zumutbar ist, um die Zugangssperren effektiver zu machen.

\footnotetext{
${ }^{15}$ OGH 24.10.2017, 4 Ob 121/17y, BitTorrent, MR 2017, 317 (Daum) = ecolex 2018/75, 161 (Zemann).

${ }^{16}$ Gegenständlich können Access-Provider erst nach einer Abmahnung auf Unterlassung geklagt werden (vergleiche $\$ 81$ Abs 1a UrhG); siehe OGH 24.06.2014, 4 Ob 71/14s, UPC Telekabel II, ÖB1 2014/50, 237 (Anzenberger) = MR 2014, 201 (Walter) sowie Kraft, Zugangssperren zu Webseiten als Mittel der Rechtsdurchsetzung, MR 2014, 171.

${ }^{17}$ Nach EuGH 27.03.2014, C-314/12, UPC Telekabel I, MR 2014, 82, sind das Grundrecht auf Schutz des geistigen Eigentums, das Grundrecht auf unternehmerische Freiheit sowie das Grundrecht auf freie Meinungsäußerung und Informationsfreiheit gegeneinander abzuwägen.

${ }^{18}$ Es müssen auch qualitative Merkmale berücksichtigt werden, so etwa, ob die rechtmäßigen Inhalte nur auf den gesperrten Seiten vorhanden sind oder auch auf anderen Seiten.

${ }^{19}$ In diesem Zusammenhang soll auch der als Lockmittel dienende Name The Pirate Bay eine Rolle spielen.

${ }^{20} \mathrm{OGH}$ 24.01.2018, 3 Ob 1/18w, Impugnationsklage bei Sperverfügungen/UPC Telekabel III, MR 2018, 74 (Walter $)=$ ecolex 2018/232, 540 (Woller) .
} 
Zum Sachverhalt: Aufgrund einer Einstweiligen Verfügung musste ein AccessProvider den Zugang zu kinox.to und movie4k.to sperren. Der Access-Provider kam dieser Vorgabe mit einer DNS-Sperre ${ }^{21}$ nach. Das heißt: Gaben seine Kunden kinox. to oder movie4k.to als Browser-URL ein, verhinderte der Access-Provider, dass diese Seiten angezeigt wurden.

Über den Provider wurde daraufhin eine Geldstrafe von 6000 Euro verhängt, weil er nur eine DNS-Sperre und keine IP-Sperre ${ }^{22}$ eingerichtet hatte. Der Provider hatte entsprechend nicht dafür gesorgt, dass die Server-IP-Adressen von kinox.to und movie4k.to als solche vom Datenverkehr im eigenen Netzwerk ausgeschlossen wurden.

Der Access-Provider wehrte sich mit einer exekutionsrechtlichen Impugnationsklage. Er machte geltend, dass er sehr wohl alle zumutbaren Maßnahmen getroffen habe.

Der OGH sieht das anders. Für den Senat ist ausschlaggebend, dass der Provider nicht alles Mögliche und ihm Zumutbare ausschöpfte, um den Zugang zu kinox.to und movie4k.to zu sperren. Eine IP-Sperre wäre technisch möglich und auch von den Kosten her nicht unverhältnismäßig gewesen. Außerdem hätte sich die Exekutionsbewilligung nur auf zwei Websites und damit nur auf zwei IP-Adressen bezogen.

Damit sind neben DNS-Sperren auch IP-Sperren für Access-Provider zumutbar und exekutierbar.

\section{EuGH C-484/14, McFadden/Sony ${ }^{23}$}

Als Betreiber eines Ladengeschäfts bot Herr McFadden Kunden und Passanten unentgeltlich und anonym Zugang zu seinem WLAN an. Dieses WLAN war bewusst nicht mit einem Passwort gesichert, weil die Öffentlichkeit unmittelbaren Zugang zum Internet haben sollte.

Über dieses offene WLAN wurde ein urheberrechtlich geschütztes Werk rechtswidrig öffentlich zur Verfügung gestellt. Daraufhin mahnte Sony Herrn McFadden ab. Gegen diese Abmahnung erhob Herr McFadden negative Feststellungsklage, und zwar mit der Begründung, für Rechtsverletzungen Dritter nicht zu haften.

\footnotetext{
${ }^{21}$ Server für das Domain Name Service (DNS) werten Domainnamen von Websites aus und ,übersetzen“ diese URLs in IP-Adressen (beispielsweise wird http://www.uibk.ac.at zu 138.232.17.233). Sperren die Access-Provider die DNS-Einträge in den DNS-Servern, erhalten ihre Nutzer im Zuge der Anfrage keine IP-Adresse.

${ }^{22}$ Sperren die Access-Provider bestimmte IP-Adressen von Servern im Internet (zum Beispiel 138.232.17.233) und schließen sie vom Datenverkehr im eigenen Netzwerk aus, so können ihre Kunden die Inhalte nicht mehr aus dem Internet abrufen.

${ }^{23}$ EuGH 15.09.2016, C-484/14, McFadden/Sony, ÖB1 2017/14, 49 (Handig) = ecolex 2016/480, 1089 (Woller) sowie Kresbach/Greiner, Keine Haftung des Betreibers eines ungeschützten WLAN-Netzes für Urheberrechtseingriffe Dritter, MR 2016, 238.
} 
Ausgangspunkt für die Beurteilung der Haftung ist gegenständlich die E-Commerce-RL. ${ }^{24}$ Diese beschränkt die Haftung - unter anderem - für von Dritten begangene Rechtsverletzungen, wenn drei Voraussetzungen kumulativ erfüllt sind. Der Access-Provider (hier: WLAN-Provider) darf

1. die Übermittlung nicht veranlasst haben,

2. den Empfänger der Übertragung nicht ausgewählt haben und

3. die übermittelten Informationen nicht ausgewählt oder verändert haben.

Sind diese drei Voraussetzungen erfüllt, haften WLAN-Provider nicht. Folgerichtig haftet auch Herr McFadden nicht für fremde Urheberrechtsverletzungen, wenn diese über sein offenes WLAN begangen werden. Anders gewendet: Der Rechteinhaber hat keinen Anspruch auf Schadenersatz gegen einen WLANBetreiber, wenn Dritte das WLAN für Urheberrechtsverletzungen benutzen. ${ }^{25}$

Dem ungeachtet kann auf gerichtlichen Antrag eines Rechteinhabers hin der Anbieter eines offenen WLAN sehr wohl dazu verpflichtet werden, Urheberrechtsverletzungen vorzubeugen beziehungsweise diese abzustellen. ${ }^{26}$ Laut EuGH muss ein WLAN durch ein Passwort gesichert werden ${ }^{27}$ und müssen die Nutzer zusätzlich um das erforderliche Passwort zu erhalten - ,ihre Identität offenbaren, damit sie nicht anonym handeln können“" ${ }^{28}$

\section{OGH 4 Ob 81/17s, Bild des Wilderers/Lichtbild in Fernsehsendung ${ }^{29}$}

Zum Sachverhalt: Der beklagte Privatfernsehsender strahlte eine Reportage über einen bekannten Soziologen und Kulturforscher aus. In dieser Sendung wurde auch über einen Osttiroler Wilderer berichtet, der vor mehr als 35 Jahren von einem Jäger erschossen worden war. Hervorhebung fand vor allem der Bruder des Wilderers und

\footnotetext{
${ }^{24}$ Access-Provider, die der Öffentlichkeit unentgeltlich ein WLAN zur Verfügung stellen, um die Aufmerksamkeit potenzieller Kunden auf die angebotenen Waren beziehungsweise Dienstleistungen zu lenken, erbringen einen Dienst der Informationsgesellschaft im Sinn der E-Commerce-RL (Richtlinie 2000/31/EG des Europäischen Parlaments und des Rates vom 8. Juni 2000 über bestimmte rechtliche Aspekte der Dienste der Informationsgesellschaft, insbesondere des elektronischen Geschäftsverkehrs, im Binnenmarkt, ABl L 178 vom 17.07.2000, 1).

${ }^{25} \mathrm{Da}$ der Rechteinhaber keinen Anspruch auf Schadenersatz hat, kann er auch keine Erstattung der für sein Schadenersatzbegehren aufgewendeten Abmahn- oder Gerichtskosten verlangen.

${ }^{26}$ Grundsätzlich muss der WLAN-Anbieter die Wahl haben, welche Maßnahmen er ergreift.

${ }^{27}$ Die E-Commerce-RL schließt ausdrücklich Maßnahmen aus, die auf eine Überwachung der durch beziehungsweise über ein Kommunikationsnetz übermittelten Informationen abzielen.

${ }^{28}$ Der EuGH weist in diesem Zusammenhang auch darauf hin, dass insbesondere Passwörter einen Abschreckungseffekt haben.

${ }^{29}$ OGH 26.09.2017, 4 Ob 81/17s, Bild des Wilderers/Lichtbild in Fernsehsendung, ÖB1-LS 2018/9, $26($ Handig $)=$ MR 2017, 325 (Walter $)$.
} 
dessen Aussagen, wobei im Lauf der Reportage ein Lichtbild des Wilderers mindestens 13 Mal eingeblendet wurde, und zwar ohne den Fotografen zu nennen.

Der Rechtsschutzverband der (Berufs-)Fotografen Österreichs klagte. Vor dem OGH war nur mehr der Unterlassungsanspruch strittig, welcher bejaht wurde. ${ }^{30}$

Der OGH prüft zum einen $\S 42 \mathrm{e}$ UrhG, das unwesentliche Beiwerk, welches mit der Urheberrechts-Novelle $2015^{31}$ eingeführt wurde. Ein solches unwesentliches Beiwerk liegt aber nur vor, wenn einem Gegenstand noch weniger als geringe oder untergeordnete Bedeutung zukommt - falls also ein Bild auch weggelassen oder ausgetauscht werden kann, ohne dass die Wirkung im Ganzen ${ }^{32}$ beeinflusst wird. ${ }^{33}$

$\rightarrow$ Wenn das Lichtbild des Wilderers in der Reportage mindestens 13 Mal eingeblendet wird, so ist das nicht beiläufig oder zufällig. In diesem Fall erfüllt das Lichtbild einen dramaturgischen Zweck, es ist nicht beliebig austauschbar und daher kein unwesentliches Beiwerk.

Zum anderen wurde im Zuge der Urheberrechts-Novelle 2015 auch die Zitatfreiheit umgestaltet. Vorliegend hilft aber $§ 42 \mathrm{fUrhG}$ nicht weiter. Ein zulässiges Bildzitat muss sich nämlich mit dem übernommenen Bild auseinandersetzen. Das heißt: Zitate brauchen einen Zitatzweck, weil es der Zitatzweck ist, der bestimmt, ob und in welchem Umfang zitiert werden darf. ${ }^{34}$ Solche Zitatzwecke wären zum Beispiel die Belehrung, Begründung oder die Erläuterung der eigenen Darstellung.

$\rightarrow$ Zeigt man das Lichtbild des Wilderers mehrfach, ohne sich inhaltlich mit dem Bild auseinanderzusetzen, dann ist das ein bloßes Aufhübschen oder Illustrieren der Reportage. Zulässiger Zitatzweck liegt jedenfalls keiner vor und damit auch kein zulässiges Bildzitat.

Daher besteht der Unterlassungsanspruch des klagenden Rechtsschutzverbands zu Recht.

\footnotetext{
${ }^{30}$ Der klagende Rechtsschutzverband forderte ursprünglich Unterlassung, Schadenersatz (Schadenspauschalierung in der Höhe des doppelten angemessenen Entgelts) und Urteilsveröffentlichung.

${ }^{31}$ Bundesgesetz, mit dem das Urheberrechtsgesetz und das Verwertungsgesellschaftengesetz 2006 geändert werden (Urheberrechts-Novelle 2015 - Urh-Nov 2015), BGBl I Nr 99/2015 vom 13.08.2015.

${ }^{32}$ In Bezug auf die Gesamtwirkung des Hauptgegenstands.

${ }^{33}$ Ein unwesentliches Beiwerk darf weder stil- noch stimmungsbildend wirken; es darf nicht charakteristisch sein, keine bestimmte Wirkung haben und auch keine Aussage unterstreichen.

${ }^{34}$ Ferner ist zu prüfen, ob der Zitatzweck auch anders erreicht werden kann, beispielsweise indem man den Rechteinhaber um Zustimmung fragt.
} 
Open Access Dieses Kapitel wird unter der Creative Commons Namensnennung 4.0 International Lizenz (http://creativecommons.org/licenses/by/4.0/deed.de) veröffentlicht, welche die Nutzung, Vervielfältigung, Bearbeitung, Verbreitung und Wiedergabe in jeglichem Medium und Format erlaubt, sofern Sie den/die ursprünglichen Autor(en) und die Quelle ordnungsgemäß nennen, einen Link zur Creative Commons Lizenz beifügen und angeben, ob Änderungen vorgenommen wurden.

Die in diesem Kapitel enthaltenen Bilder und sonstiges Drittmaterial unterliegen ebenfalls der genannten Creative Commons Lizenz, sofern sich aus der Abbildungslegende nichts anderes ergibt. Sofern das betreffende Material nicht unter der genannten Creative Commons Lizenz steht und die betreffende Handlung nicht nach gesetzlichen Vorschriften erlaubt ist, ist für die oben aufgeführten Weiterverwendungen des Materials die Einwilligung des jeweiligen Rechteinhabers einzuholen. 